\title{
Physical fitness and its association with cognitive performance in Chilean schoolchildren: The Cogni-Action Project
}

\author{
Patricio Solis-Urra $^{1,2}$ (1) | Javier Sanchez-Martinez ${ }^{1,3}$ | Jorge Olivares-Arancibia ${ }^{1,4}$ | \\ Jose Castro Piñero $^{5,6}{ }^{\circ}$ ｜ Kabir P. Sadarangani ${ }^{7,8}$ | Gerson Ferrari ${ }^{9}$ | Fernando \\ Rodríguez-Rodríguez $^{1}$ ๑ | Anelise Gaya ${ }^{10}$ | Camila Felin Fochesatto ${ }^{10}$ | Carlos Cristi-Montero ${ }^{1}$
}

${ }^{1}$ IRyS Group, Physical Education School, Pontificia Universidad Católica de Valparaíso, Valparaíso, Chile

${ }^{2}$ PROFITH "PROmoting FITness and Health through Physical Activity" Research Group, Department of Physical Education and Sport, Faculty of Sport Sciences, University of Granada, Granada, Spain

${ }^{3}$ Escuela de Kinesiología, Facultad de Salud, Universidad Santo Tomás, Viña del Mar, Chile

${ }^{4}$ Grupo AFySE, Investigación en Actividad Física y Salud Escolar, Escuela de Pedagogía en Educación Física, Facultad de Educación, Universidad de las Américas, Santiago, Chile

${ }^{5}$ GALENO Research Group, Department of Physical Education, Faculty of Education Sciences, University of Cádiz, Puerto Real, Spain

${ }^{6}$ Biomedical Research and Innovation Institute of Cádiz (INiBICA) Research Unit, Cádiz, Spain

${ }^{7}$ Universidad Autónoma de Chile, Chile

${ }^{8}$ Escuela de Kinesiología, Facultad de Salud y Odontología, Universidad Diego Portales, Santiago, Chile

${ }^{9}$ Escuela de Ciencias de la Actividad Física, el Deporte y la Salud, Universidad de Santiago de Chile (USACH), Chile

${ }^{10}$ Projeto Esporte Brasil (PROESP-Br), School of Physical Education, Physiotherapy and Dance, Post-graduation Program in Human Movement Sciences, Federal University of Rio Grande do Sul, Porto Alegre, Brazil

\section{Correspondence}

Carlos Cristi-Montero, IRyS Group,

Physical Education School, Pontificia

Universidad Católica de Valparaíso,

Valparaíso, Chile.

Email: carlos.cristi.montero@gmail.com

Funding information

The "Cogni-Action Project" is supported by the National Commission for Scientific and Technological Research CONICYT/ FONDECYT INICIACION 2016 grant no. 11160703. Patricio Solis-Urra was supported by a grant from the National Agency for Research and Development (ANID)/BECAS Chile/72180543. This work is part of a Ph.D. Thesis conducted in the Doctoral Programme in Biomedicine of the University of Granada, Spain.
This study aimed to establish the association and differences in a diversity of cognitive domains according to cardiorespiratory fitness (CRF), muscular fitness (MF), and speed-agility fitness (S-AF) level in a large sample of Chilean schoolchildren. 1171 Chilean schoolchildren aged 10-14 years participated. CRF, MF, and S-AF were assessed through the ALPHA-fitness test battery. Cognition was evaluated through the NeuroCognitive Performance Test, which involved eight tests related to four main domains: cognitive flexibility (CF), working memory (WM), inhibitory control (IC), and intelligence (IN). Both global (multivariate) and individual (univariate) analyses were performed to determine the differences in cognitive functioning according to low-, middle-, and high-fitness level. The global analyses showed a significant main effect for CRF, $F_{(16,940)}=3.08, p \leq .001$ and MF groups, $F_{(16,953)}=2.30, p=.002$, but not for S-AF, $F_{(16,948)}=1.37, p=.105$. CRF shows a significant main effect in seven of eight tests, involving CF, WM, IC, and IN domains, whereas MF shows a significant main effect in five of eight tests without association with IN. SA-F shows a significant main effect only with IC. Statistical differences were found between the low- and middle/high-fitness groups but not between the middle- and high-fitness groups. At a global level, both CRF and MF seem to be associated with a higher cognitive profile in scholars; however, at an individual level, all fitness components show 
a favorable relationship to some cognitive domine. Then, future cognitive developing strategies should consider all fitness components, prioritizing those low-fitness schoolchildren.

\section{K E Y W O R D S}

adolescents, cognition, healthy lifestyle, muscle strength, physical fitness, schools

\section{1 | INTRODUCTION}

Growing scientific evidence supports that physical fitness is a potent marker of brain health throughout the life span. ${ }^{1}$ Overall, systematic reviews have shown a strong relationship between physical fitness components such as cardiorespiratory fitness (CRF), muscular fitness (MF), and speed-agility fitness (S-AF) with cognitive function, academic achievement, and brain health in children and adolescents. ${ }^{2-4}$ Indeed, having a high-fitness level may enhance both children's behavioral and biological aspects, transferring these benefits to the cognitive and educational field. ${ }^{5}$

Cardiorespiratory fitness has been the most studied component of physical fitness. To date, there is strong evidence displaying that children with a higher CRF level present greater levels of attention, working memory, and cognitive flexibility, and in turn, higher academic achievement. ${ }^{1-3}$ Interestingly, even though some evidence exist on the association between MF and S-AF with academic achievement, ${ }^{6}$ their relation to diverse cognitive domains is less consistent or unexplored. ${ }^{7,8}$ At this moment and based on the current literature, it is possible to speculate that CRF, SA-F, and MF could be related to cognitive domains into a differentiated extent. ${ }^{1,9,10}$ This idea considers that their underlying mechanisms, although they have not been identified completely, would not necessarily be the same. ${ }^{1,10}$ For instance, CRF seems to elicit a beneficial impact on neurogenesis, cognitive function, and brain metabolism throughout a complex sequence of physiological events linked to the release of neurotrophic factors, which supports the existence of a musclebrain endocrine loop. ${ }^{9}$ However, the MF influence on the brain has been more specifically related to an enhanced neuromuscular and motor system properties. ${ }^{10}$

In addition to the above mentioned, there are still other concerns and gaps to cover in this matter; thereby, the present study focuses on two of them. On the one hand, cognition is a complex mental process which is fundamental to acquiring knowledge and achieve personal goals, and several cognitive domains form it. ${ }^{11,12}$ For this reason, it is appropriated that cognition will be evaluated throughout a set of cognitive tasks in order to provide a more comprehensive approach and to generates more tailed public health and educational recommendations in this knowledge area. In this sense, this study supports the idea of exploring how each physical fitness component is related to a wide variety of cognitive abilities and domains.

On the other hand, it is possible to find scarce evidence studying the relationship between physical fitness and cognition in the Latin-American children population. ${ }^{13}$ This gap not only is relevant to consider because most of the evidence published on this matter come from developed countries and thereby involve a more favorable social and educational contexts ${ }^{13}$; but also because it is well known that social vulnerability exerts a substantial and detrimental effect on children's brain health, cognition, and school achievement. ${ }^{14-16}$ Besides, at the current rate, by 2030 , around $63 \%$ of the world's children will be living in lower-middle-income nations like the most Latin-American countries. ${ }^{17}$ In this way, nowadays, exploring factors helping to resolve this global concern is essential considering also the impact of the COVID-19 pandemic on the rate of poverty in this region. ${ }^{18}$ Therefore, this study aimed to establish the association and differences in a diversity of cognitive tests and domains according to the level of CRF, MF, and S-AF in a large sample of Chilean schoolchildren.

\section{2 | METHODS}

\section{1 | Study design}

This study is part of the Cogni-Action Project, which seeks to establish the associations of physical activity, sedentarism, and physical fitness with brain structure and function, cognitive performance, and academic achievement in Chilean schoolchildren. ${ }^{19}$ This project was approved by the Ethics Committee of the Pontificia Universidad Católica de Valparaíso (BIOEPUCV-H103-2016) and was retrospectively registered (8/July/2020) in the Research Registry (ID: researchregistry5791). In all aspects, this research has been conducted according to the Declaration of Helsinki. Written consents have been obtained before participation from the school principal, parents, and assent from participants.

This cross-sectional study was carried out from March 2017 to October 2019, where children and adolescents in grades 5th to 8th were recruited from the public, voucher, and private schools in the Valparaiso region, Chile. This study was prepared according to the STROBE guidelines 
(Strengthening the Reporting of Observational Studies in Epidemiology) for cross-sectional studies. ${ }^{20}$

\section{2 | Study population}

Total sample size and power calculations were based on the total enrollment of schoolchildren in the Valparaiso region (5th to 8th grades) indicated by the Chilean Ministry of Education in the year 2016 (universe $N=951$ 962). It was considered an alpha error of $5 \%$, confidence interval of $99 \%$, heterogeneity of $50 \%$, and a $20 \%$ dropout. Hence, a total of 797 participants were necessary to reach a representative sample size from the second most populated region in Chile.

The general inclusion criteria were girls and boys from 5th to 8th grades (10-14 years old), and for ethical reasons, children who present any physical, psychiatric, and/ or psychological disability were also included in this project study if both children and parents approve their participation, through the signing of assent and consent, respectively. Finally, 1586 schoolchildren were involved in this project due to the elevated participation and minimal exclusion criteria to maximize diversity in social, biological, and environmental influences. For this study, 1171 schoolchildren were included after applying the following exclusion criteria: (a) being out of the stipulated age range, (b) missed the cognitive evaluation or (c) not having data on any of the variables involved for this study. In this sample, were also included 111 children who participated in a national program to improve their learning level.

\section{3 | Measurements}

All assessments took place at schools, considering two sessions of four hours each separated by eight days apart. In the first session, a complete cognitive battery and anthropometric measurements were assessed, while during the second session, physical fitness was evaluated. Trained instructors from our research team performed all evaluations, and schoolchildren had a brief familiarization trial before each test.

\section{4 | Physical fitness assessment}

Physical fitness was evaluated through the well-documented ALPHA-fitness test battery. ${ }^{21}$ Briefly, this time-efficient and low-cost fitness battery was developed to provide a set of valid, reliable, feasible, and safe field-based fitness tests in children and adolescents. It permits evaluating and monitoring a large number of children simultaneously. The ALPHAfitness test battery presents three slightly different versions which depend on the available time to administer the tests; in this study, we have used the extended version that includes all main physical fitness components (CRF, MF, and S-AF) ${ }^{21}$ Tests were performed in sports fields or indoor gym during mornings (between 9:30 and 12:00), suggesting appropriate sportswear. Verbal instructions on how to perform each test and a brief demonstration of the technique were carried out to ensure the optimal test performance. Children practiced each test previously and then started when they felt secure.

\subsection{1 | Cardiorespiratory fitness}

Cardiorespiratory fitness was evaluated with the 20-m shuttle run test and carried out at the end of the evaluation session. ${ }^{21}$ Groups of between 8 and 10 children were located at the starting line, and a sound signal indicated the run rhythm, which started at $8.5 \mathrm{~km} / \mathrm{h}$ and increased $0.5 \mathrm{~km} / \mathrm{h}$ every minute. Thus, children had to run $20 \mathrm{~m}$ and wait on the second line until the next sound signal. To ensure a progressive increase and a correct adaptation to the test, a physical education teacher ran beside children guiding the first two minutes of the test. The test ended voluntarily when the child was fatigued or unable to reach the line twice. Total time (in seconds) and the number of completed stages were registered, as recommended. ${ }^{22}$ Thus, a $z$-score of the total time (s) based on sex and age was created as a normalized CRF score.

\subsection{2 | Muscular fitness}

Upper and lower limb strength were evaluated as indicators of MF. ${ }^{21}$ On the one hand, upper limb strength was assessed by the maximum handgrip strength test using a dynamometer (Jamar Plus+ Digital Hand Dynamometer, Sammons Preston). The dynamometer was previously adjusted to the child's hand size, allowing for measures of $0-90 \mathrm{~kg}$, with a $0.1 \mathrm{~kg}$ precision. This test was performed twice (both hands), in a standing position with a fully extended elbow, and the maximum score between measures was used. Then, in order to create a relative measure of upper limb strength, the score was divided by body weight.

On the other hand, the lower limb strength was assessed through the standing long jump test. A starting line was fixed on the floor, and children had to stand with their feet parallel behind the line. At the verbal signal, children had to jump as far as possible starting with and landing on both feet at the same time. This test was performed twice (with at least 1-min rest between them), and the longest jump was recorded in centimeters $(\mathrm{cm})$. Finally, the MF score was created based on the sum of the sex- and age-standardized values of handgrip/ weight and standing long jump. 


\subsubsection{Speed-agility fitness}

Speed-agility fitness was assessed using the $4 \times 10$-m shuttle run test. ${ }^{21}$ This test accounts for speed of movement, agility, and coordination. Two lines ( $5 \mathrm{~m}$ long) separated by $10 \mathrm{~m}$ were fixed on the floor, and two cones were located in each line. Children had to run as fast as possible, taking a cloth located $\sim 50 \mathrm{~cm}$ after the first line and carrying it to the next line where they had to swap for a second cloth before running to the final line. The test was performed twice, and the fastest time was recorded in seconds. Time was multiplied by -1 , so a higher score indicated better performance. Finally, a $z$-score base on sex and age was created as a normalized S-AF score.

\subsection{Cognitive performance}

The NeuroCognitive Performance Test (NCPT) from Lumos Labs, Inc. was used to assess the children's cognitive performance. ${ }^{23}$ The NCPT has demonstrated adequate reliability and validity as a measure of cognitive performance, and good concordance with pencil-paper assessments. ${ }^{23}$ It is a brief, repeatable, web-based platform to measure several cognitive domains, including working memory, visuospatial memory, psychomotor speed, fluid and logical reasoning, response inhibition, numerical calculation, and selective and divided attention.

The NCPT was applied in schoolrooms, in groups of 25 children, each one with a laptop. The entire session lasted around one hour, which consisted of a brief explanation about the session's aim, a demonstration and practice before each test, and finally the execution. Children's answers were resolved before starting each cognitive test. Table 1 displays a summary of all eight cognitive tests assessed in the NCPT. More details about tests are elsewhere. ${ }^{19,23}$ In order to facilitate results description and its discussion, we grouped the cognitive test into four domains based on literature and also the main cognitive ability of each task, in summary: (a) Cognitive flexibility (TMT-A, TMT-B, and digit symbol coding tests), (b) Working memory (forward and reverse memory span tests), (c) Inhibitory control (go/no-go test), and (d) Intelligence (problem-solving and progressive matrices tests) (details in Table 1). Despite the aforementioned, all analyses were individually performed to explore differences depending on fitness level and in order to detect any possible cognitive particularity. Each test was scaled following a normal inverse transformation of the percentile rank. ${ }^{23}$ These procedures provide the benefit of having scaled scores derived on the same normal distribution with a mean of 100 and a standard deviation of 15 .

\section{6 | Covariates}

Sex, school administration, peak height velocity (PHV), and body mass index (BMI) were used as covariates in all
TA B LE 1 Domains and tests from The NeuroCognitive Performance Test

\begin{tabular}{|c|c|c|c|}
\hline Domain & $\begin{array}{l}\text { Cognitive } \\
\text { tests }\end{array}$ & $\begin{array}{l}\text { Cognitive } \\
\text { functions involved }\end{array}$ & $\begin{array}{l}\text { Graphical } \\
\text { representation }\end{array}$ \\
\hline \multirow[t]{3}{*}{$\mathrm{CF}$} & $\begin{array}{l}\text { Trail } \\
\text { making } \\
\text { test A }\end{array}$ & $\begin{array}{l}\text { Attention, cognitive } \\
\text { flexibility, and } \\
\text { processing speed }\end{array}$ & 3 \\
\hline & $\begin{array}{l}\text { Trail } \\
\text { making } \\
\text { test B }\end{array}$ & $\begin{array}{l}\text { Attention, cognitive } \\
\text { flexibility, and } \\
\text { processing speed }\end{array}$ & $\rightarrow 2$ \\
\hline & $\begin{array}{l}\text { Digit } \\
\text { symbol } \\
\text { coding }\end{array}$ & Processing speed & $\begin{array}{l}* \\
?\end{array}$ \\
\hline \multirow[t]{2}{*}{ WM } & $\begin{array}{l}\text { Forward } \\
\text { memory } \\
\text { span }\end{array}$ & $\begin{array}{l}\text { Visual short-term } \\
\text { memory }\end{array}$ & \\
\hline & $\begin{array}{l}\text { Reverse } \\
\text { memory } \\
\text { span }\end{array}$ & Working memory & \\
\hline IC & $\begin{array}{l}\text { Go/no-go } \\
\text { test }\end{array}$ & $\begin{array}{l}\text { Inhibitory control } \\
\text { and processing } \\
\text { speed }\end{array}$ & \\
\hline \multirow[t]{2}{*}{ IN } & $\begin{array}{r}\text { Balance } \\
\text { scale }\end{array}$ & $\begin{array}{l}\text { Quantitative and } \\
\text { analogical } \\
\text { reasoning }\end{array}$ & $\int_{0}^{1}$ \\
\hline & $\begin{array}{r}\text { Progressive } \\
\text { matrices }\end{array}$ & $\begin{array}{l}\text { Problem-solving } \\
\text { and fluid } \\
\text { reasoning/ } \\
\text { intelligence }\end{array}$ & 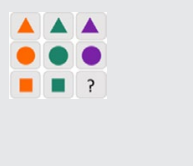 \\
\hline
\end{tabular}

Abbreviations: CF, cognitive flexibility; IC, inhibitory control; IN, intelligence; WM, working memory.

models. These covariates were chosen by previous evidence indicating its influence on cognitive performance, ${ }^{1-3,6,7}$ and also based on our own exploratory analysis (using a stepwise regression approach) in this sample. Sex was included due to diverse differences between boys and girls on the main topic of this study. ${ }^{24}$ The school administration (public, voucher, or private) was included as a covariate due to their strong association with cognitive, physical fitness, and academic performance. ${ }^{15,16}$ School administration is a close indicator of socioeconomic and parental education levels in the Chilean context. ${ }^{25}$ PHV was calculated as a maturity status indicator. ${ }^{26}$ It was computed by subtracting the PHV age from the chronological age. The difference in years was defined as a value of maturity offset. Finally, the weight was measured with a digital balance in which precision and maximum weight were of 0.1 and $150 \mathrm{~kg}$, respectively (OMROM, HN289-LA), while height with a portable stadiometer (SECA, model 213, GmbH). Then, the BMI z-score was calculated using World Health Organization 2007 growth reference for school-aged children. ${ }^{27}$ 


\section{7 $\quad$ Statistical analysis}

Descriptive analyses are presented as means, standard deviation, frequency, and percentage. Quantitative variables were checked for normal distribution using the Kolmogorov-Smirnov test. Differences between boys and girls in continuous and factor variables were tested using the Student's $t$ test for equal variances and the Chi-square test, respectively. Physical fitness was grouped into low (corresponding to quartile 1), middle (corresponding to quartile 2- quartile 3), and high (corresponding to quartile 4), as previously used in this field. ${ }^{28}$ To reduce the chances of Type I error from multiple comparisons and to test the "global main effect," three multivariate analyses of covariance (MANCOVA) were conducted investigating group differences (low-, middle-, and high-fitness level), using the percentile score of the eight cognitive tests as a continuous outcome, and each fitness variable (CRF, MF, and S-AF) as an explanatory factor, including sex, school administration, PHV, and BMI as covariates. Whereas to test the "individual main effect," one-way analysis of covariance (ANCOVA) was performed to assess mean differences between fitness level groups in each cognitive test, including the same covariates mentioned above in the models. Finally, post hoc pairwise comparisons were conducted to establish differences in the marginal estimated means in each pair of groups (lowvs. middle-, low- vs. high-, and middle- vs. high-fitness level), and $p$-values were corrected using Tukey contrasts. Additionally, effect size (ES) estimation was calculated using emmeans package in R. Consequently, the ES was interpreted as no effect $(<0.2)$, small $(0.2<0.5)$, medium $(0.5<0.8)$, and large $(\geq 0.8) .{ }^{29}$ Finally, as the interaction by sex was not significant $(p>.1)$, all analyses are presented together (boys and girls). Assumptions of linearity, normality, multicollinearity, and homoscedasticity were plotted, inspected, and verified. All analyses used complete case data, and no imputation was carried out. All statistical analyses were performed in $\mathrm{R}$ (version 3.6.1; $\mathrm{R}$ Foundation for Statistical Computing), and statistical significance was set at $p<.05$.

\section{3 | RESULTS}

Table 2 presents the main participant characteristics. Significant differences were found in PHV, weight, CRF, SLJ, HGS, S-AF.

TABLE 2 Main characteristics of the participants

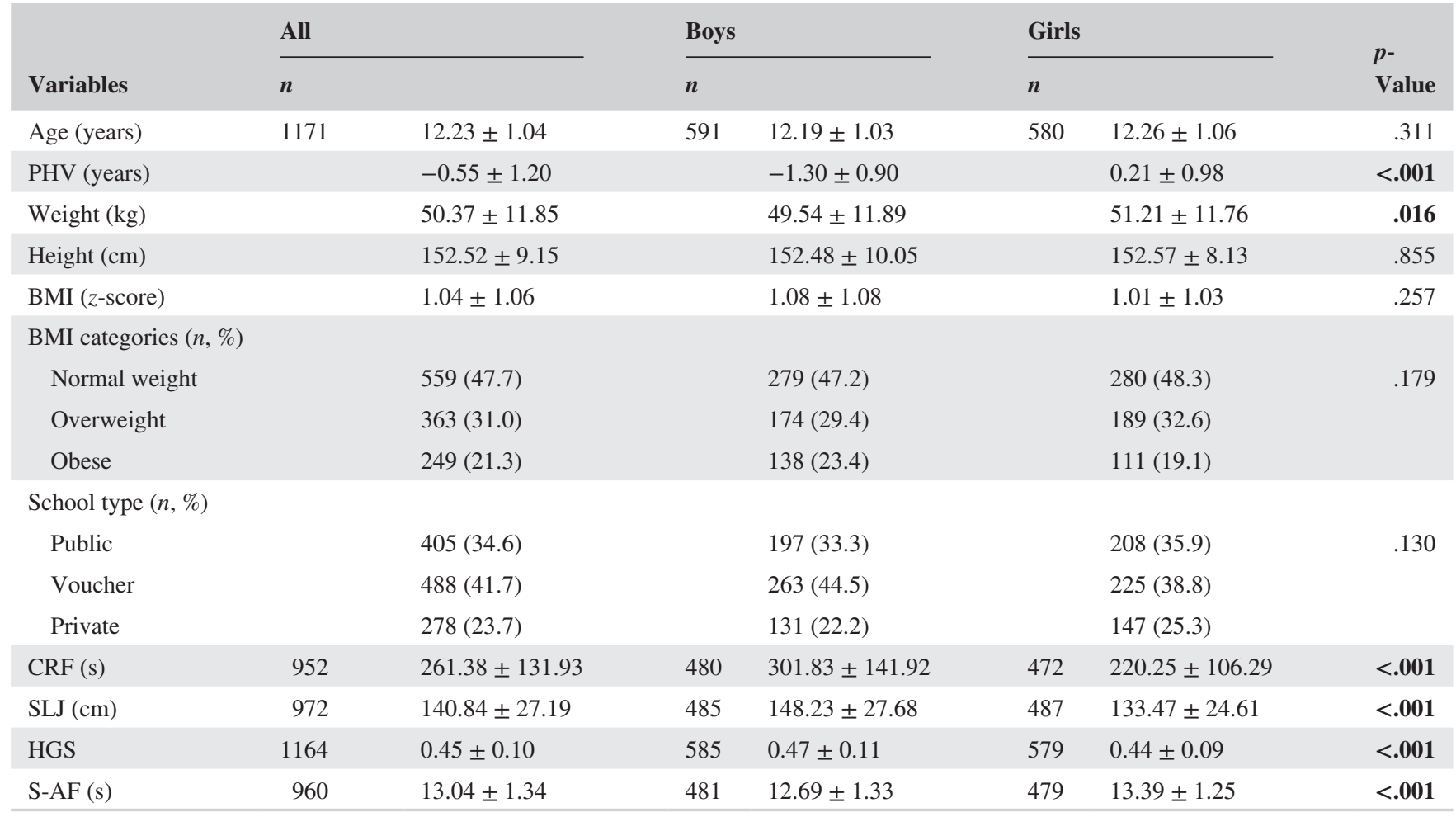

Note: Values are presented as mean $\pm \mathrm{SD}$ or frequency and percentage. Values in bold indicate significant differences. $p$-Value corresponding to $t$-test between boys and girls.

Abbreviations: BMI, body mass index (BMI-for-age Z-score and categories were calculated using WHO 2007 growth reference) ${ }^{27}$; CRF, cardiorespiratory fitness (measured by the 20-m shuttle run test); handgrip (kg)/body weight (kg)); HGS, handgrip strength (relative values; PHV, peak height velocity offset; S-AF, speedagility fitness (measure by the $4 \times 10-\mathrm{m}$ shuttle run test); SLJ, Standing long jump. 
Figure 1 shows a significant main effect on CRF and MF fitness components (Figure 1A, $p<.001$; and Figure 1B, $p=.002$, respectively), but not on $\mathrm{S}-\mathrm{AF}$ ( $p=.105$, Figure 1C). Additionally, Figure 1 shows significant main differences in seven $\left(p^{\prime} \mathrm{s} \leq .021\right)$, five ( $p$ 's $\leq .020)$, and two ( $p$ 's $\leq .023)$ cognitive tests according to $\mathrm{CRF}, \mathrm{MF}$, and S-AF components, respectively.

Table 3 shows analyses of pairwise comparisons and ES according to CRF groups. There were significant differences between low vs. middle and low vs. high groups in cognitive flexibility (trail making test A and B, and digit symbol coding), working memory (forward and reverse memory span), inhibition control (go/no-go), and intelligence domains (progressive matrices) (all $p$ 's $\leq .035$ ). All of these differences presented a small ES ( $d=0.204-0.446)$. Furthermore, only a difference was found between the low vs. middle group in the trail making test $\mathrm{B}(p=.014$ and $d=0.230)$, as well as between low versus high groups in the go/no-go test $(p=.001$ and $d=0.346$ ). No differences were found between middle versus high groups in the rest of the tests. Also, no difference among groups was observed in the problem-solving test.

Table 4 displays analyses of pairwise comparisons and ES according to MF groups. There were significant differences between low versus middle and low versus high groups in one test from working memory and inhibition control domains (forward memory span and go/no-go test, respectively) (all $p$ 's $\leq .34$ ). All of these associations presented a small ES ( $d=0.204-0.373)$. Furthermore, only differences between low versus middle groups were found in five tests, corresponding to cognitive flexibility (trail making test A and digit symbol coding), working memory (reverse and forward memory span), and inhibition control domains (go/no-go test) (all $p$ 's $\leq .034, d=0.204-0.373$ ). No differences were found between middle vs. high groups in the remaining tests.

Table 5 shows analyses of pairwise comparisons and ES according to S-AF groups. There were significant differences between low versus middle and low versus high groups only in the working memory domain (reverse memory span test, $p$ 's $\leq .046 ; d=0.217-0.224)$. Furthermore, only a difference between low versus high groups was found in the inhibition control domain (go/no-go test, $p=.014, d=0.263$ ). No differences were found between middle vs. high groups in the rest of the tests.

\section{4 | DISCUSSION}

The present study aimed to determine the association and differences in diverse cognitive domains according to groups of CRF, MF, and S-AF levels in a large sample of Chilean schoolchildren. Our analysis indicates that CRF and MF, but not S-AF presented a global significant main effect on cognitive performance. At an individual level, CRF was associated with the four cognitive domains analyzed (cognitive
Low Middle High fitness level

\section{(A) Cardiorespiratory fitness}

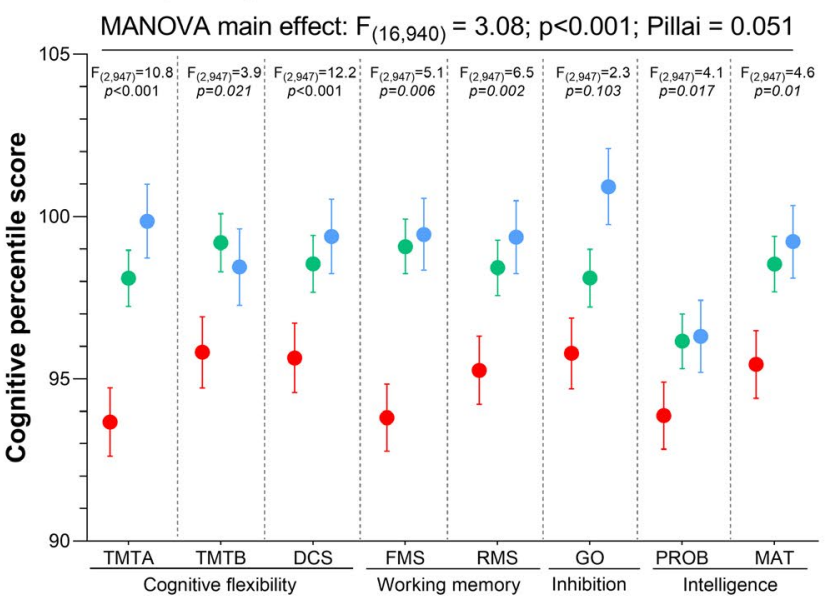

(B) Muscular fitness

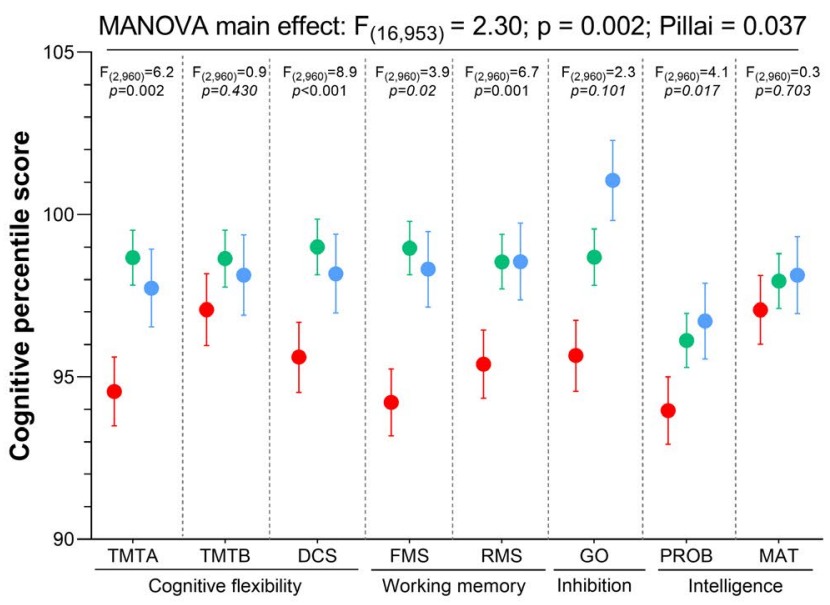

(C) Speed/Agility fitness

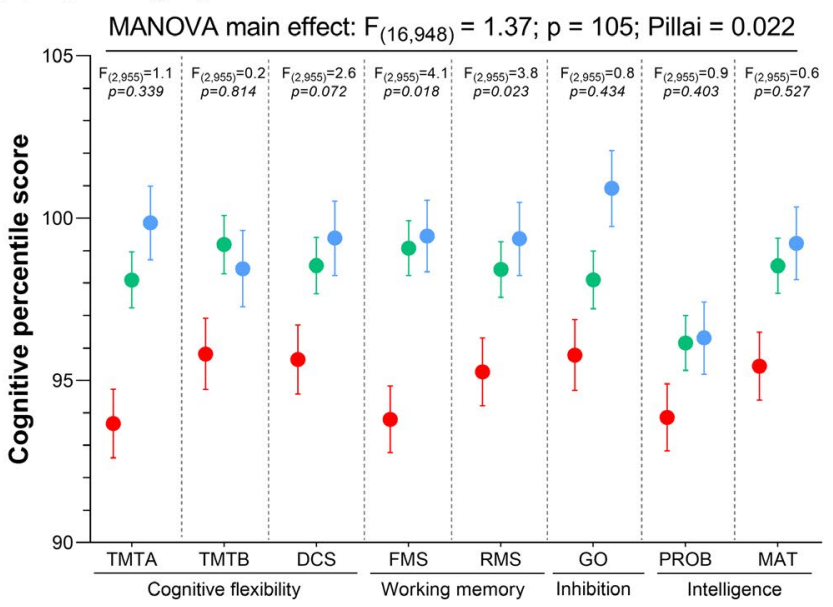

F I G U RE 1 Multivariate cognitive effect according to each fitness component. DSC, Digit symbol coding; FWS, Forward memory span; GO, go/no-go; MAT, Progressive matrices; PROB, Problem-solving; RMS, Reverse memory span; TMT-A, Trail making test A; TMT-B, Trail making test $\mathrm{B}$

flexibility, working memory, inhibition control, and intelligence), while MF was associated with three cognitive 
T A B L E 3 Pairwise comparison, according to cardiorespiratory fitness groups for each cognitive task

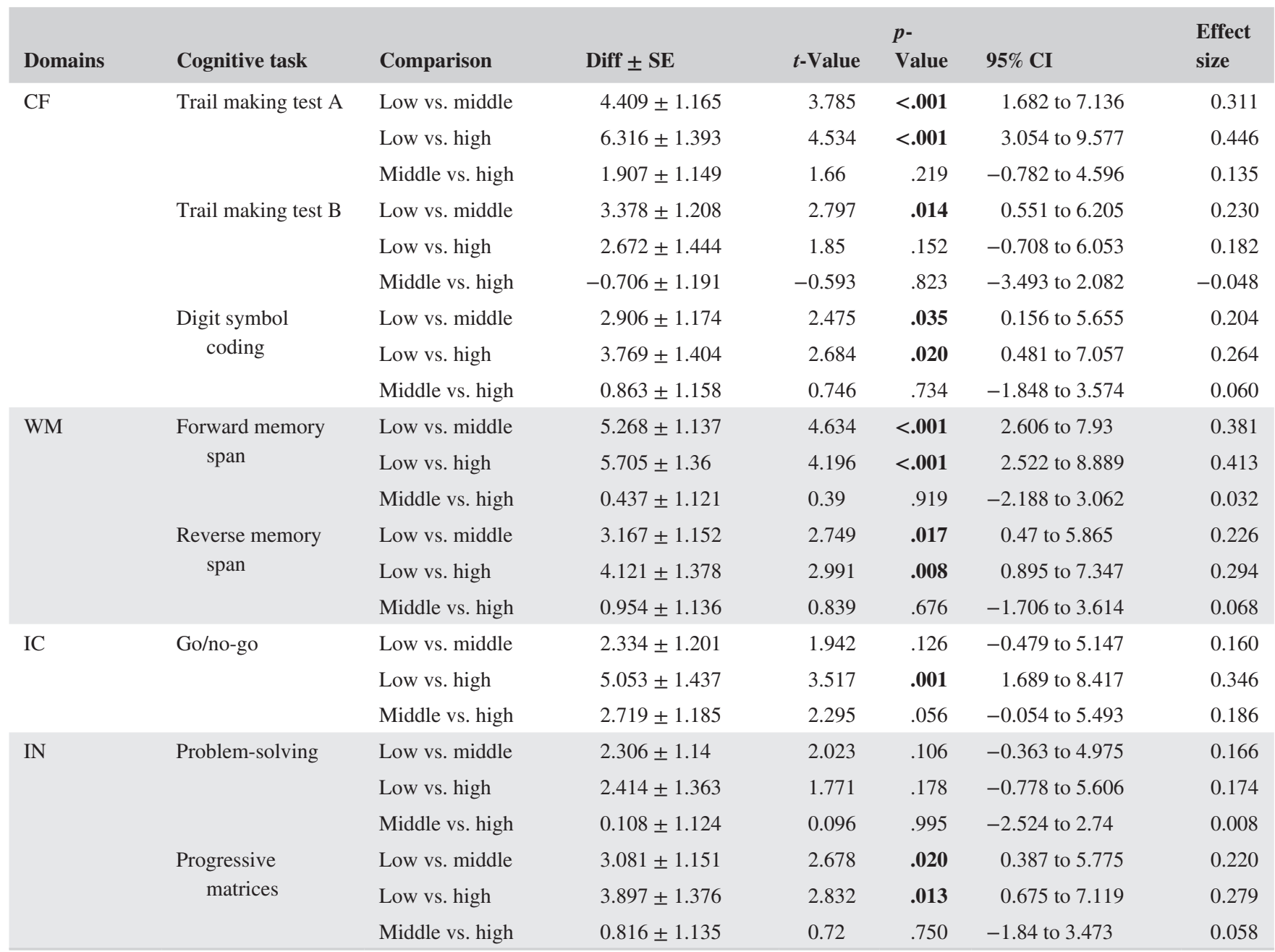

Note: Values in bold indicate significant differences and effect size $\geq 0.20$ (small effect).

Abbreviations: CF, cognitive flexibility; CI, confidence interval; Diff, difference of marginal mean value of ANCOVA adjusted for peak height velocity, sex, school type, and body mass index; Effect size by Cohen; IC, inhibitory control; IN, intelligence; $p$-value adjusted for multiple comparisons using Tukey contrasts; SE, Standard error; WM, working memory.

domains (cognitive flexibility, working memory, and inhibition control), and finally, S-AF was associated with two cognitive domains (working memory and inhibition control). Moreover, differences were observed principally between low and middle or high physical fitness groups, but not between the middle and high group. Interestingly, reverse memory spam and go/no-go tests, which involved cognitive abilities such as working memory, inhibitory control, and processing speed (linked to executive function), were associated with all three fitness components.

Several systematic reviews and meta-analyses support the main findings of this study. ${ }^{4,15}$ Our results reveal that CRF has a strong association both at a global and individual level with all cognitive tests except with the problem-solving test. Despite this unique not significant association, CRF was linked to at least one test within the four cognitive domains. These findings are in line with the vast literature in developing countries showing that CRF is the most significant component of physical fitness related to attention capacity, ${ }^{7,30,31}$ cognitive flexibility, ${ }^{4}$ working memory, ${ }^{30}$ inhibitory control, ${ }^{32}$ and intelligence. ${ }^{32}$ Multiple underlying bases explain the beneficial CRF impact on cognition, including molecular mechanisms, and structural and functional brain outcomes. ${ }^{1,33}$ However, as MF and SA-F have been investigated scarcely, the effect and plausible mechanisms of each one on the brain remain unclear yet.

Regarding MF, our findings show a significant association both at a global and individual level. MF was related to cognitive flexibility (trail making test A and digit symbol coding), working memory (forward and reverse memory span), and inhibition control (go/no-go). Previous studies have found a relation between MF and working memory; nonetheless, this association seems to be dependent on CRF. ${ }^{7,8}$ In children with overweight or obesity, the association of MF with the cognitive flexibility and planning ability shows a borderline association, while 
T A B L E 4 Pairwise comparison, according to muscular fitness groups for each cognitive task

\begin{tabular}{|c|c|c|c|c|c|c|c|}
\hline Domains & Cognitive task & Comparison & $\operatorname{Diff} \pm S E$ & $t$-Value & $\begin{array}{l}p \text { - } \\
\text { Value }\end{array}$ & 95\% CI & $\begin{array}{l}\text { Effect } \\
\text { size }\end{array}$ \\
\hline \multirow[t]{7}{*}{$\mathrm{CF}$} & \multirow[t]{2}{*}{ Trail making test A } & Low vs. middle & $4.192 \pm 1.167$ & 3.592 & .001 & 1.462 to 6.922 & 0.295 \\
\hline & & Middle vs. high & $-1.03 \pm 1.172$ & -0.878 & .651 & -3.772 to 1.713 & -0.072 \\
\hline & \multirow[t]{2}{*}{ Trail making test $\mathrm{B}$} & Low vs. middle & $1.599 \pm 1.21$ & 1.322 & .379 & -1.231 to 4.43 & 0.108 \\
\hline & & Middle vs. high & $-0.523 \pm 1.216$ & -0.431 & .902 & -3.367 to 2.32 & -0.036 \\
\hline & \multirow{3}{*}{$\begin{array}{l}\text { Digit symbol } \\
\text { coding }\end{array}$} & Low vs. middle & $3.415 \pm 1.182$ & 2.89 & .011 & 0.65 to 6.179 & 0.237 \\
\hline & & Low vs. high & $2.599 \pm 1.462$ & 1.778 & .175 & -0.821 to 6.019 & 0.181 \\
\hline & & Middle vs. high & $-0.816 \pm 1.187$ & -0.687 & .768 & -3.593 to 1.961 & -0.057 \\
\hline \multirow{3}{*}{ WM } & \multirow{3}{*}{$\begin{array}{l}\text { Reverse memory } \\
\text { span }\end{array}$} & Low vs. middle & $3.165 \pm 1.155$ & 2.74 & .017 & 0.463 to 5.868 & 0.225 \\
\hline & & Low vs. high & $3.185 \pm 1.429$ & 2.228 & .066 & -0.159 to 6.528 & 0.226 \\
\hline & & Middle vs. high & $0.019 \pm 1.161$ & 0.017 & 1.000 & -2.696 to 2.735 & 0.001 \\
\hline \multirow[t]{3}{*}{ IC } & \multirow[t]{3}{*}{ Go/no-go } & Low vs. middle & $2.98 \pm 1.199$ & 2.485 & .034 & 0.175 to 5.785 & 0.204 \\
\hline & & Low vs. high & $5.442 \pm 1.483$ & 3.668 & .001 & 1.972 to 8.912 & 0.373 \\
\hline & & Middle vs. high & $2.462 \pm 1.205$ & 2.044 & .101 & -0.356 to 5.279 & 0.169 \\
\hline \multirow[t]{3}{*}{ IN } & \multirow[t]{3}{*}{ Problem-solving } & Low vs. middle & $2.141 \pm 1.138$ & 1.881 & .142 & -0.522 to 4.804 & 0.154 \\
\hline & & Low vs. high & $2.785 \pm 1.408$ & 1.977 & .116 & -0.51 to 6.079 & 0.201 \\
\hline & & Middle vs. high & $0.644 \pm 1.144$ & 0.563 & .838 & -2.032 to 3.319 & 0.046 \\
\hline
\end{tabular}

Note: Values in bold indicate significant differences and effect size $\geq 0.20$ (small effect).

Abbreviations: CF, cognitive flexibility; CI, confidence interval; Diff, difference of marginal mean value of ANCOVA adjusted for peak height velocity, sex, school type, and body mass index; Effect size by Cohen; IC, inhibitory control; IN, intelligence; $p$-value adjusted for multiple comparisons using Tukey contrasts; SE, standard error; WM, working memory.

no association was observed with inhibitory control. ${ }^{32}$ In the case of Latin-American children, the MF was favorably associated with attention capacity, but this result was moderate by their fatness level. ${ }^{34}$ Contrary, MF was not associated with attention capacity or cognitive control in European ${ }^{7}$ and Australian ${ }^{8}$ adolescents, respectively. The last mentioned also supports one of the gaps in this research scenario regarding the apparent differences among countries and ethnicities due to their social and economic disparities. ${ }^{14,15}$ Finally, even though it is possible to appreciate divergences respect to the relation between MF and cognitive functions, our results provide novel evidence that, in the present population, there is a clear association between them.

Concerning S-AF, this component was significantly associated only at an individual level with working memory and inhibitory control (reverse memory span and go/ no-go test, respectively). Similar findings were found in
Switzerland pre-schoolers, indicating a significant relationship between S-AF with memory and attention performance. ${ }^{35}$ In Latin-American children, all fitness components were associated with children's attention capacity. ${ }^{34}$ In general, although the evidence that addresses $\mathrm{MF}$ and $\mathrm{S}-\mathrm{AF}$ is more limited and inconsistent than CRF, some studies show a positive association between S-AF and MF with higher gray matter volumes in diverse brain regions. ${ }^{6}$ As occur with CRF, these findings between S-AF and MF with brain matter volumes might support a better cognitive profile in children and adolescents. ${ }^{1}$

Overall, our results support the favorable association between three physical fitness components and cognitive performance in children and adolescents of an underexplored Latin-American country, which contributes to the geographical gap on this research area. ${ }^{13}$ Besides, we observe a small ES in most of the significant association between fitness components and cognition tests; however, at a public health 
TA B L E 5 Pairwise comparison, according to speed-agility groups for each cognitive task

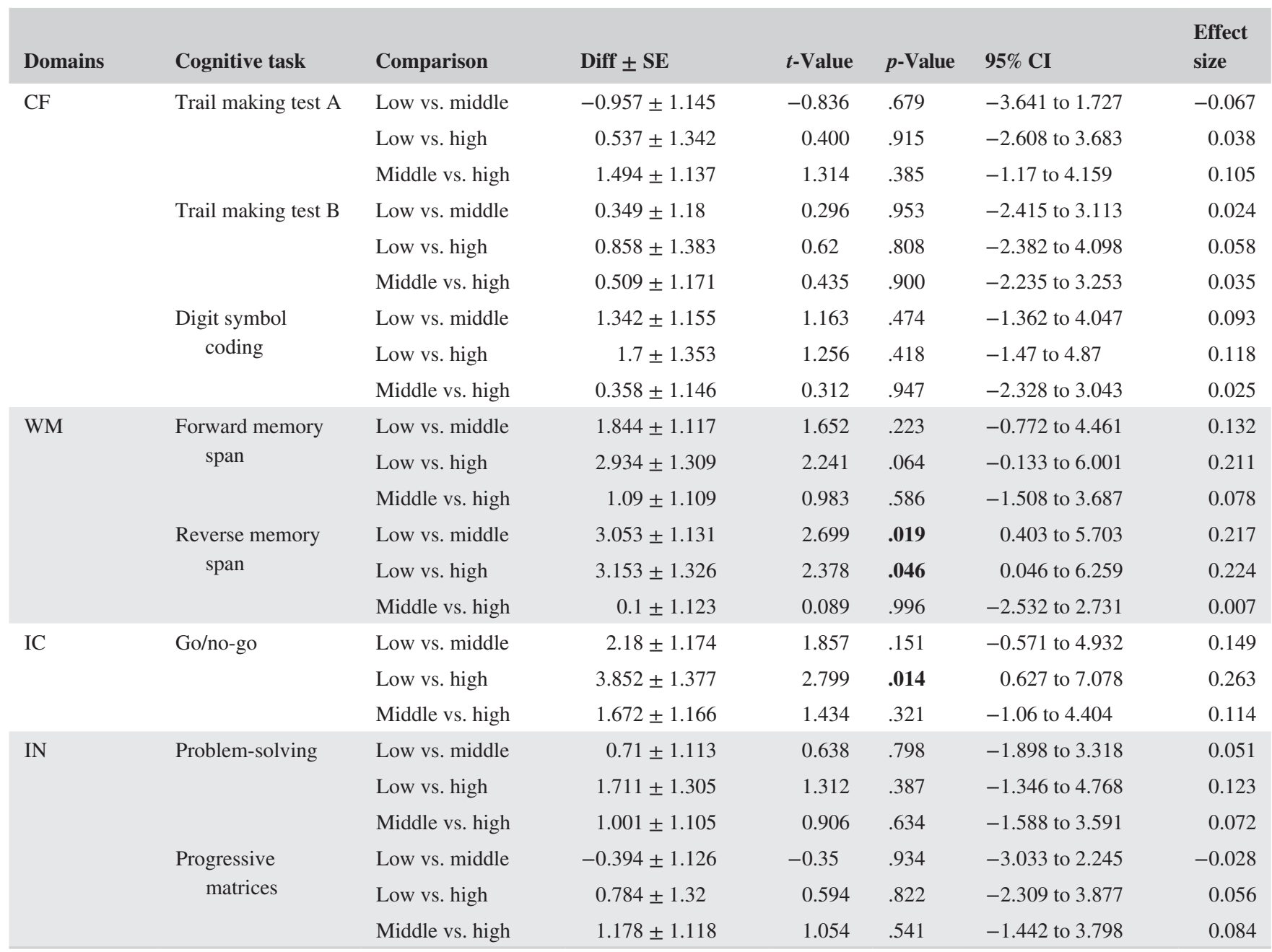

Note: Values in bold indicate significant differences and effect size $\geq 0.20$ (small effect).

Abbreviations: CF, cognitive flexibility; CI, confidence interval; Diff, difference of marginal mean value of ANCOVA adjusted for peak height velocity, sex, school type, and body mass index; Effect size by Cohen; IC, inhibitory control; IN, intelligence; $p$-value adjusted for multiple comparisons using Tukey contrasts; SE, standard error; WM, working memory.

and educational context, even a small effect is meaningful when considering that each cognitive capacity could be affected (positively and negatively) by a vast multitude of internal and external factors (eg, sleep quality, fatness, social vulnerability, and others). ${ }^{1,16,34}$

Finally, it is essential to highlight that the degree of association between a specific cognitive domain would depend on the brain relationship with each fitness component. Thereby, we could speculate based on the scientific literature that the CRF influence on the brain would be linked to the increased physiological response to cover the oxygen and energy exercise demand. ${ }^{1,33,36}$ In contrast, MF would be associated with a direct neuromuscular mechanism boosting the strength and power demand, ${ }^{10,37}$ and $\mathrm{S}-\mathrm{AF}$ would be related to the high demand of coordination and a mix between power strength and aerobic capacity. ${ }^{38,39}$ Thus, increasing the level of children' CRF, MF, and S-AF, and not focusing in only one fitness component, could play an essential role related to an enhanced global cognition performance, which consequently may help to improve both academic achievement and brain health in children and adolescents. ${ }^{1-3,7,11,40}$ Intervention studies are needed to corroborate the last mentioned.

\section{1 $\quad$ Strengths and limitations}

Strengths of the present work include that, to the best of our knowledge, this is the first study in Latin-America using a large sample of schoolchildren exploring the association between different physical fitness components and several cognitive domains. Besides, both keys variables in this study, fitness and cognition, were evaluated using an extensive set of physical and cognitive tests, giving a general and not a 
particular view of their relationship. Finally, our results were significant and consistent even adjusted to several covariates that present a strong association with cognition (sex, school administration, PHV, and BMI). However, the principal study limitation is that the Cogni-Action Project employed a cross-sectional design, precluding inferences about causality.

\section{5 | CONCLUSION}

In conclusion, children and adolescents with higher fitness levels present a superior global and individual cognitive performance. Also, each fitness component showed a differentiated association degree with each cognitive task and domain. Therefore, both children and adolescents with the lowest level of fitness, and all three fitness components (CRF, MF, and $\mathrm{S}-\mathrm{AF}$ ), not just one, must be considered as interventional targets associated with a better cognitive profile in LatinAmerican schoolchildren. Intervention studies are needed to corroborate our findings and establish the independent influence of each fitness component over cognition.

\section{CONFLICT OF INTERESTS}

The authors declare to have no competing interests.

\section{AUTHORS' CONTRIBUTIONS}

CC-M designed the research; CC-M, PS-U, JS-M, and JO-A conducted research; CC-M and PS-U analyzed data, CC-M and PS-U wrote the paper; JCP, KPS, GLMF, FR-R, AG, and CFF critically reviewed the manuscript; CC-M had primary responsibility for final content. All authors provided critical feedback on the manuscript and read and approved the final manuscript.

\section{DATA AVAILABILITY STATEMENT}

The data that support the findings of this study are available on request from the corresponding author. The data are not publicly available due to privacy or ethical restrictions.

\section{ORCID}

Patricio Solis-Urra (1D https://orcid. org/0000-0002-2493-9528

Jose Castro Piñero (D) https://orcid. org/0000-0002-7353-0382

Fernando Rodríguez-Rodríguez (i) https://orcid. org/0000-0002-4999-4857 Carlos Cristi-Montero (1D https://orcid. org/0000-0002-9940-507X

\section{REFERENCES}

1. Stillman CM, Esteban-Cornejo I, Brown B, Bender CM, Erickson KI. Effects of exercise on brain and cognition across age groups and health states. Trends Neurosci. 2020;43(7):533-543.
2. Van Waelvelde $H$, Vanden Wyngaert $K$, Mariën $T$, Baeyens D, Calders P. The relation between children's aerobic fitness and executive functions: a systematic review. Infant and Child Development. 2020;29(3):e2163.

3. Lubans D, Richards J, Hillman C, et al. Physical activity for cognitive and mental health in youth: a systematic review of mechanisms. Pediatrics. 2016;138(3):e20161642.

4. Fedewa AL, Ahn S. The effects of physical activity and physical fitness on children's achievement and cognitive outcomes: a metaanalysis. Res Q Exerc Sport. 2011;82(3):521-535.

5. Mavilidi MF, Drew R, Morgan PJ, Lubans DR, Schmidt M, Riley N. Effects of different types of classroom physical activity breaks on children's on-task behaviour, academic achievement and cognition. Acta Paediatr. 2020;109(1):158-165.

6. Esteban-Cornejo I, Tejero-González CM, Martinez-Gomez D, et al. Independent and combined influence of the components of physical fitness on academic performance in youth. J Pediatr. 2014;165(2):306-312 e302.

7. Cadenas-Sanchez C, Vanhelst J, Ruiz JR, et al. Fitness and fatness in relation with attention capacity in European adolescents: the HELENA study. J Sci Med Sport. 2017;20(4):373-379.

8. Shigeta TT, Leahy AA, Smith JJ, Eather N, Lubans DR, Hillman $\mathrm{CH}$. Cardiorespiratory and muscular fitness associations with older adolescent cognitive control: fitness associations with adolescent cognitive control. J Sport Health Sci. 2021;10(1):82-90.

9. Pedersen BK. Physical activity and muscle-brain crosstalk. Nat Rev Endocrinol. 2019;15(7):383-392.

10. Shaughnessy KA, Hackney KJ, Clark BC, et al. A narrative review of handgrip strength and cognitive functioning: bringing a new characteristic to muscle memory. J Alzheimers Dis. 2020(Preprint);73(4):1265-1278.

11. Esteban-Cornejo I, Tejero-Gonzalez CM, Sallis JF, Veiga OL. Physical activity and cognition in adolescents: a systematic review. J Sci Med Sport. 2015;18(5):534-539.

12. Gomez-Pinilla F, Hillman C. The influence of exercise on cognitive abilities. Compr Physiol. 2013;3(1):403-428.

13. Wassenaar TM, Williamson W, Johansen-Berg H, et al. A critical evaluation of systematic reviews assessing the effect of chronic physical activity on academic achievement, cognition and the brain in children and adolescents: a systematic review. Int J Behav Nutr Phys Act. 2020;17(1):79.

14. Caldas SJ, Reilly MS. The mediating influence of physical activity levels on 3rd-grade academic achievement. J Res Child Educ. 2019;33(2):271-289.

15. Hanushek EA, Woessmann L. How much do educational outcomes matter in OECD countries? Econ Policy. 2011;26(67):427-491.

16. Ursache A, Noble KG, Pediatric Imaging $\mathrm{N}$, Genetics $\mathrm{S}$. Socioeconomic status, white matter, and executive function in children. Brain Behav. 2016;6(10):e00531.

17. UNICEF. Every Child Learns: UNICEF Education Strategy 20192030. New York, NY: UNICE; 2019.

18. Zar HJ, Dawa J, Fischer GB, Castro-Rodriguez JA. Challenges of COVID-19 in children in low- and middle-income countries. Paediatr Respir Rev. 2020;35:70-74.

19. Solis-Urra P, Olivares-Arancibia J, Suarez-Cadenas E, et al. Study protocol and rationale of the "Cogni-action project" a crosssectional and randomized controlled trial about physical activity, brain health, cognition, and educational achievement in schoolchildren. BMC Pediatr. 2019;19(1):260. 
20. von Elm E, Altman DG, Egger M, et al. The Strengthening the Reporting of Observational Studies in Epidemiology (STROBE) statement: guidelines for reporting observational studies. J Clin Epidemiol. 2008;61(4):344-349.

21. Ruiz JR, Castro-Pinero J, Espana-Romero V, et al. Field-based fitness assessment in young people: the ALPHA health-related fitness test battery for children and adolescents. Br J Sports Med. 2011;45(6):518-524.

22. Tomkinson GR, Lang JJ, Léger LA, et al. Response to criticisms of the $20 \mathrm{~m}$ shuttle run test: deflections, distortions and distractions. Br J Sports Med. 2019;53(19):1200-1201.

23. Morrison GE, Simone CM, Ng NF, Hardy JL. Reliability and validity of the NeuroCognitive Performance Test, a web-based neuropsychological assessment. Front Psychol. 2015;6:1652.

24. Resaland GK, Moe VF, Bartholomew JB, et al. Gender-specific effects of physical activity on children's academic performance: the active smarter kids cluster randomized controlled trial. Prev Med. 2018;106:171-176.

25. Carrasco A, Gunter HM. The "private" in the privatisation of schools: the case of Chile. Educational Review. 2019;71(1):67-80.

26. Moore SA, Mckay HA, Macdonald H, et al. Enhancing a somatic maturity prediction model. Med Sci Sports Exerc. 2015;47(8):1755-1764.

27. Onis MD, Onyango AW, Borghi E, Siyam A, Nishida C, Siekmann J. Development of a WHO growth reference for school-aged children and adolescents. Bull World Health Organ. 2007;85:660-667.

28. Visier-Alfonso ME, Sanchez-Lopez M, Martinez-Vizcaino V, Jimenez-Lopez E, Redondo-Tebar A, Nieto-Lopez M. Executive functions mediate the relationship between cardiorespiratory fitness and academic achievement in Spanish schoolchildren aged 8 to 11 years. PLoS One. 2020;15(4):e0231246.

29. Cohn J. Statistical Power Analysis for the Behavioral Sciences. Hillsdale, NJ: Lawrence Earlbam Associates; 1988.

30. Mora-Gonzalez J, Esteban-Cornejo I, Cadenas-Sanchez C, et al. Fitness, physical activity, working memory, and neuroelectric activity in children with overweight/obesity. Scand J Med Sci Sports. 2019;29(9):1352-1363.

31. Mora-Gonzalez J, Esteban-Cornejo I, Cadenas-Sanchez C, et al. Physical fitness, physical activity, and the executive function in children with overweight and obesity. J Pediatr. 2019;208:50-56.e51.
32. Mora-Gonzalez J, Esteban-Cornejo I, Solis-Urra P, et al. Fitness, physical activity, sedentary time, inhibitory control, and neuroelectric activity in children with overweight or obesity: the ActiveBrains project. Psychophysiology. 2020;57(6):e13579.

33. Mattson MP, Moehl K, Ghena N, Schmaedick M, Cheng A. Intermittent metabolic switching, neuroplasticity and brain health Nat Rev Neurosci. 2018;19(2):63-80.

34. García-Hermoso A, Hormazábal-Aguayo I, Fernández-Vergara O, et al. Physical fitness components in relation to attention capacity in Latin American youth with overweight and obesity. Scand J Med Sci Sports. 2020;30(7):1188-1193.

35. Niederer I, Kriemler S, Gut J, et al. Relationship of aerobic fitness and motor skills with memory and attention in preschoolers (Ballabeina): a cross-sectional and longitudinal study. BMC Pediatr. 2011;11:34.

36. Cooper C, Moon HY, van Praag H. On the run for hippocampal plasticity. Cold Spring Harb Perspect Med. 2018;8(4):a029736.

37. Yao WX, Jiang Z, Li J, et al. Brain functional connectivity is different during voluntary concentric and eccentric muscle contraction. Front Physiol. 2016;7:521.

38. Koutsandreou F, Wegner M, Niemann C, Budde H. Effects of motor versus cardiovascular exercise training on children's working memory. Med Sci Sports Exerc. 2016;48(6):1144-1152.

39. Krakauer JW, Hadjiosif AM, Xu J, Wong AL, Haith AM. Motor learning. Compr Physiol. 2011;9(2):613-663.

40. Hillman $\mathrm{CH}$, Biggan JR. A review of childhood physical activity, brain, and cognition: perspectives on the future. Pediatr Exerc Sci. 2017;29(2):170-176.

How to cite this article: Solis-Urra P, SanchezMartinez J, Olivares-Arancibia J, et al. Physical fitness and its association with cognitive performance in Chilean schoolchildren: The Cogni-Action Project. Scand J Med Sci Sports. 2021;31:1352-1362. https:// doi.org/10.1111/sms.13945 